\title{
Completion of the Causal Completability Problem
}

\author{
Michał Marczyk, Leszek Wroński \\ Draft version, 4 October 2011
}

\begin{abstract}
We give a few results concerning the notions of causal completability and causal closedness of classical probability spaces (Hofer-Szabó, Rédei \& Szabó [1999], Gyenis \& Rédei [2004]). Answering a question from HoferSzabó et al. [1999], we prove that any classical probability space has a causally closed extension. We also employ the notion of causal up-to- $n$ closedness (Wroński \& Marczyk [2010a]) to show that any finite classical probability space with rational probabilities on the atoms of the event algebra can be extended to a finite space which is causally up-to-3-closed. Lastly, we prove that any classical probability space can be extended to a space in which all correlations between events which are logically independent modulo measure zero event have a countably infinite common cause system (for the definition of the latter notion, see Hofer-Szabó \& Rédei [2004]). Collectively, these results show that it is surprisingly easy to find Reichenbach-style explanations for correlations, underlining doubts as to whether this approach can yield a philosophically relevant account of causality.
\end{abstract}

\section{Contents}

1 Introduction 1

2 Basic definitions and results in the literature 2

3 Causal completability the easy way_ "splitting the atom" $\quad 7$

4 Causal completability of classical probability spaces - the general case

5 Infinite statistical common cause systems for arbitrary pairs $\quad 10$

6 Conclusion 12

A Proof of theorem 3 12

\section{Introduction}

In recent years we have seen a resurgence of the discussion of Reichenbach's Principle of the Common Cause (Reichenbach [1971]). The principle, according 
to which for any correlated events there exists a common cause satisfying certain probabilistic conditions we discuss below (see definition 5), has been extensively criticised both on the general philosophical level (see e.g. Arntzenius [1992] and Sober [2001]) and more formally, with reference to the empirically falsified Bell inequalities (see the discussion sparked by van Fraassen [1982]). This did not stop it, however, from becoming one of the main inspirations for the causal Markov condition, crucial in the field of Bayesian nets (see e.g. Pearl [2000] and Williamson [2005]). During the last decade support for the principle came from the purely mathematical direction, namely that of causal closedness and completability of probability spaces (see Hofer-Szabó et al. [1999] and Gyenis \& Rédei [2004]). It was proven e.g. that whenever a probability space contains a correlation for which no event fulfilling Reichenbach's conditions exists, the space in question can be extended to a bigger space (with the measure preserved, i.e. all 'old' events retaining their probabilities) in which such an event does exist. Of course, new correlations appear in the bigger space and the natural question arises whether this process can be stopped: can we extend the initial probability space to one containing common causes in the Reichenbachian sense for all correlations? The answer is "yes", and the argument is the proof of our theorem 2.

The remaining results of this paper, theorems 1 and 3 , concern generalisations of Reichenbach's idea to entities with similar explanatory features, called "Common Cause Systems" (see definition 6; the "usual' common cause is a special case of a common cause system), and the related notion of causal up-to- $n$ closedness (of which, again, the 'usual' notion of causal closedness is a special case). Theorem 3 demonstrates that any "interesting" correlation in an atomless probability space (i.e. one involving events neither of which is a cause of the other) has uncountably many common causes and common cause systems of any finite or countably infinite size. (Wroński \& Marczyk [2010b] shows that no uncountable common cause systems exist.) Theorem 1 shows how a reasonable degree of causal completeness can be obtained already in the setting of finite probability spaces, as long as the space to be extended involves only rational probabilities. Together with theorem 2 they show that Reichenbach-style explanations are surprisingly easy to come by, at least in appropriately extended spaces, raising questions as to the philosophical relevance of this account of causality.

\section{Basic definitions and results in the literature}

We shall start with a few basic concepts. In this paper we are dealing with classical probability spaces only, noting that some questions concerning causal closedness and completability of non-classical probability spaces remain unanswered (see e.g. Rédei \& Summers [2002]).

Definition 1 [Probability space] $A$ probability space is a triple $\langle\Omega, \mathcal{F}, P\rangle$ such that:

- $\Omega$ is a non-empty set;

- $\mathcal{F}$ is a nonempty family of subsets of $\Omega$ which is closed under complement and countable union; 
- $P$ is a function from $\mathcal{F}$ to $[0,1] \subseteq \mathbb{R}$ such that

- $P(\Omega)=1$;

- $P$ is countably additive: for a countable family $\mathcal{G}$ of pairwise disjoint members of $\mathcal{F}, P(\cup \mathcal{G})=\sum_{A \in \mathcal{G}} P(A)$.

In the context of a probability space $\langle\Omega, \mathcal{F}, P\rangle, \Omega$ is called the sample space, $\mathcal{F}$ is called the event space, and $P$ is called the probability function (or measure). The members of $\mathcal{F}$ are called events.

We will say that events $A, B \in S$ are (positively) correlated if $P(A \cap B)>$ $P(A) P(B)$.

Not all correlations demand explanation by means of a common cause; for example, correlations between a cause and its effects are typically explained by the causal relation the two events stand in. Things are different if we believe that neither of the two events causally influences the other: for example, a rise of the column of mercury in a barometer does not cause a storm. According to Reichenbach, in such cases we should expect that there exists a common cause fulfilling a few precise mathematical conditions (see definition 5 below). The starting point of the assessment whether all correlations to be explained possess common causes in such a sense is determining which correlations demand explanation; that is, defining a binary relation on the event space which is to be understood as a relation of causal independence, determined by our knowledge of the causal situation in the given context.

A minimal candidate for such a relation is that of logical independence. We are not surprised that "being blonde" and "having hair" are correlated, since one entails the other. "The coin having fallen heads up" and "the coin having fallen tails down" are perfectly correlated, simply because they are the same event. (Not) to deal with such cases, we will now give formal definitions of two relations of logical independence. Intuitively, we will regard two events as logically independent if, when we learn that one of the events occurs (or does not occur), we cannot infer that the other occurs (or does not occur), for all four Boolean combinations.

Definition 2 [Logical independence] We say that events $A, B \in S$ are logically independent $\left(\langle A, B\rangle \in L_{\text {ind }}\right)$ iff all of the following sets are nonempty:

- $A \cap B$

- $A \cap B^{\perp}$;

- $A^{\perp} \cap B$;

- $A^{\perp} \cap B^{\perp}$.

We say that events $A, B \in S$ are logically independent modulo measure zero event, or genuinely independent $\left(\langle A, B\rangle \in L_{\text {ind }}^{+}\right)$, iff all of the following numbers are positive:

- $P(A \cap B)$;

- $P\left(A \cap B^{\perp}\right)$;

- $P\left(A^{\perp} \cap B\right)$; 
- $P\left(A^{\perp} \cap B^{\perp}\right)$.

Equivalently, two events are logically independent if neither of the events is contained in the other one, their intersection is non-empty and the union of the two is properly included in the whole space. Two events are genuinely independent if every Boolean combination of them has a non-zero probability of occurring. It is always true that $L_{i n d}^{+} \subseteq L_{\text {ind }}$.

We will now provide the definitions needed to replicate the concept of a common cause Reichenbach would have us expect to exist for correlations.

Definition 3 Let $A, B \in S$. An event $C$ is said to be a screener-off for the pair $\{A, B\}$ if $P(A \cap B \mid C)=P(A \mid C) P(B \mid C)$. In the case where $A$ and $B$ are correlated we also say that $C$ screens off the correlation.

Definition 4 Let $A, B \in S$. We say that a family of events $\left\{C_{i}\right\}$ satisfies the statistical relevance condition with regard to the pair $\{A, B\}$ if whenever $i \neq j$

$$
\left(P\left(A \mid C_{i}\right)-P\left(A \mid C_{j}\right)\right)\left(P\left(B \mid C_{i}\right)-P\left(B \mid C_{j}\right)\right)>0
$$

Definition 5 [Statistical Common Cause] Let $\langle\Omega, \mathcal{F}, P\rangle$ be a probability space. Let $A, B \in \mathcal{F}$. Any event $C \in \mathcal{F}$ different from both $A$ and $B$ such that

- both $C$ and $C^{\perp}$ are screener-offs for the pair $\{A, B\}$,

- the pair $\left\{C, C^{\perp}\right\}$ satisfies the statistical relevance condition with regard to the pair $\{A, B\}$,

is called a statistical common cause $(S C C)$ of $A$ and $B$.

In part of the literature, the above concept is referred to as "Reichenbachian Common Cause" (see e.g. Hofer-Szabó \& Rédei [2004], Hofer-Szabó \& Rédei [2006]). We believe this to be unfortunate for a few reasons. The fact that an event meets some probabilistic requirements is decidedly not enough to justify it being called a "cause" of something else - this is the main reason for us putting the word "Statistical" in the name of the concept. Reichenbach himself observed that common effects may also meet the same requirements. He also did not require that all common causes have these probabilistic properties. In other words, in Reichenbach's own view the screening off and statistical relevance conditions are neither necessary nor sufficient for being a common cause. For an extended discussion of these issues, see Wroński [2010].

Statistical common causes have a few features due to which it may seem reasonable to view them as candidates for explanations of correlations. First, notice that due to screening off, conditioning on a statistical common cause renders its effects statistically independent - the correlation disappears. Second, an SCC raises the probability of occurrence of both its effects, which is an initially natural, though controversial (see Beebee [1998]), condition for causes in the context of probabilistic causation. Third, it was proved by Reichenbach already that statistical common causes have a certain deductive feature: namely, from both screening off conditions together with the statistical relevance condition it is possible to deduce the correlation between the effects of the cause.

Why should correlations be explained only by means of simple "yes"/"no" events, though? Perhaps a different, more general concept could be used, which 
would correspond to not necessarily binary traits in the population under examination. It would be ideal if the concept shared the deductive features of statistical common causes. This project was pursued by Hofer-Szabó \& Rédei [2004], who generalize the concept to the so-called "Reichenbachian Common Cause Systems"; in line with our previously outlined practice, we substitute "Statistical" for "Reichenbachian":

Definition 6 [Statistical Common Cause System] Let $\langle\Omega, \mathcal{F}, P\rangle$ be a probability space. A partition of unity of $\mathcal{F}$ is said to be a statistical common cause system (SCCS) for $A$ and $B$ if it satisfies the statistical relevance condition w.r.t. $A$ and $B$, all its members are different from both $A$ and $B$, and all its members are screener-offs for the pair.

The cardinality of the partition is called the size of the statistical common cause system.

In the sequel we will sometimes say that a probability space contains an SCCS, which means that the SCCS is a partition of unity of the underlying algebra of the space. We also sometimes use the expression "common cause (system)" instead of "statistical common cause (system)" to shorten the formulation of some arguments.

As far as explanatory power is concerned, SCCSs fare similarly to SCCs: this is more or less evident with regard to the screening off and statistical relevance conditions, and it was shown in Hofer-Szabó \& Rédei [2004] that existence of an SCCS for events $A, B \in S$ entails a correlation between those events.

The following definition is a refinement of the $\mathrm{SCC}(\mathrm{S})$ idea, expressing the requirement that a common cause (all elements of a common cause system) should be meaningfully different from both correlated events.

Definition 7 [Proper $S C C(S)$ ] A statistical common cause $C$ of events $A$ and $B$ is a proper statistical common cause of $A$ and $B$ if it differs from both $A$ and $B$ by more than a measure zero event. It is an improper $S C C$ of these events otherwise.

An SCCS $\left\{C_{i}\right\}_{i \in I}$ of events $A$ and $B$ is a proper SCCS of $A$ and $B$ if all its elements differ from both $A$ and $B$ by more than a measure zero event. It is an improper SCCS of these events otherwise.

In the current paper we will show (Theorem 3) that any correlation between genuinely independent events in an atomless classical probability space has a proper infinite SCCS.

Should someone prefer it, the following definition could be phrased in terms of SCCSs only.

Definition 8 [Causal up-to-n-closedness] We say that a classical probability space is causally up-to- $n$-closed w.r.t. to a relation of independence $R_{\text {ind }}$ if all pairs of correlated events independent in the sense of $R_{\text {ind }}$ possess a proper statistical common cause or a proper statistical common cause system of size at most n.

If the space is causally up-to-2-closed, we also say that it is causally closed or common cause closed. 
The next definition to be introduced is that of an atomless probability space. It turns out that such spaces are extremely rich in statistical common causes (see fact 11).

Definition 9 [Atomless probability space] A probability space $\langle\Omega, \mathcal{F}, P\rangle$ is atomless if for any $C \in \mathcal{F}$, if $P(C)>0$, then there exists $D \in \mathcal{F}$ such that $D \subseteq C$ and $0<P(D)<P(C)$.

Remarkably, this definition suffices to prove the following fact:

Fact 10 If $C$ is an event of positive probability $r$ in an atomless probability space $\langle\Omega, \mathcal{F}, P\rangle$, then for any such that $0<s<r$ there exists an event $D \in \mathcal{F}$ such that $D \subseteq C$ and $P(D)=s$.

See e.g. p. 46 of Fremlin [2001] for a proof.

We will now give all the results from the literature which we will need to refer to in the course of proving theorems 1-3.

The first fact concerns the aforementioned richness of atomless spaces:

Fact 11 (Gyenis \& Rédei [2004]) All atomless probability spaces are causally closed.

The second one says that in a finite probability space with the uniform measure, all correlations between logically independent events have a statistical common cause or a statistical common cause systems of size 3 .

Fact 12 (Wroński \& Marczyk [2010a]) Any finite classical probability space $\langle\Omega, \mathcal{F}, P\rangle$ with $P$ being the uniform measure is causally up-to-3-closed w.r.t. $L_{\text {ind }}$.

To state the next required fact properly, we need the definition of an extension of a probability space. The notion may be thought of as representing an occasion on which first a small space is constructed which corresponds to some (perhaps empirically) given probabilities, and which displays some unexplained correlations; then, in the course of seeking explanation for them, more factors are taken into account, which translates to a finer partition of the original sample space, or simply to a widening of the event space. Of course, the new space also has to match the (empirically) given probabilities.

Definition 13 [Extension] Let $\mathfrak{A}=\langle\Omega, \mathcal{F}, P\rangle$, be a probability space. A space $\mathfrak{A}^{\prime}=\left\langle\Omega^{\prime}, \mathcal{F}^{\prime}, P^{\prime}\right\rangle$ is called an extension of $\mathfrak{A}$ if there is a Boolean algebra embedding $h: \mathcal{F} \rightarrow \mathcal{F}^{\prime}$ which preserves the measure, that is, $\forall A \in \mathcal{F}, P^{\prime}(h(A))=$ $P(A)$.

In Hofer-Szabó, Rédei \& Szabó [1999] the authors discuss causal completability with regard to a family of correlated pairs of events. Their main result regarding classical probability spaces follows, in a slightly different formulation. Here, as in the original formulation, no independence relations are involved.

Definition 14 [Causal completability] Suppose $\langle\Omega, \mathcal{F}, P\rangle$ is a probability space and $\mathcal{U}$ is a family of pairs of correlated events which do not have an $S C C$ in $\langle\Omega, \mathcal{F}, P\rangle$. The space $\langle\Omega, \mathcal{F}, P\rangle$ is causally completable with regard to the family $\mathcal{U}$ if there exists an extension $\left\langle\Omega^{\prime}, \mathcal{F}^{\prime}, P^{\prime}\right\rangle$ of $\langle\Omega, \mathcal{F}, P\rangle$ by means of a homomorphism $h$ which contains an $S C C$ for $\langle h(A), h(B)\rangle$ for every pair $\langle A, B\rangle \in \mathcal{U}$. 
Fact 15 (Propos. 2 from Hofer-Szabó, Rédei \& Szabó [1999]) Every classical probability space is causally completable with regard to any finite family of correlated events.

Hofer-Szabó et al. [1999] pose the problem whether classical probability spaces are causally completable with regard to infinite families of correlated events. This we answer in the positive in section 4 .

Of course, an extension of a given space which provides explanations for some correlations may very well introduce new unexplained correlations. The extension constructed by Hofer-Szabó et al. [1999] is not expected to be causally closed, or causally up-to- $n$-closed, for any natural number $n$. For a single unexplained correlation, the extension is made from two copies of the initial space (for details, see p. 391-392 of Hofer-Szabó et al. [1999]). In the next section we will present a simple method of extending probability spaces to spaces which are causally up-to-3-closed. The method will however be restricted to finite spaces with rational probabilities on the atoms of the event space.

\section{Causal completability the easy way-"splitting the atom"}

In this section the sample spaces of the probability spaces involved will be irrelevant, so we will treat probability spaces as pairs $\langle\mathcal{F}, P\rangle, \mathcal{F}$ being a finite Boolean algebra (the spaces considered will be finite) and $P$ being a classical measure on $\mathcal{F}$.

If in a finite probability space with some non-uniform measure the atoms have rational probabilities, we can "split" them into pieces in order to arrive at a space with the uniform measure which will be an obvious, intuitive extension of the initial space. Consider the following illustration:

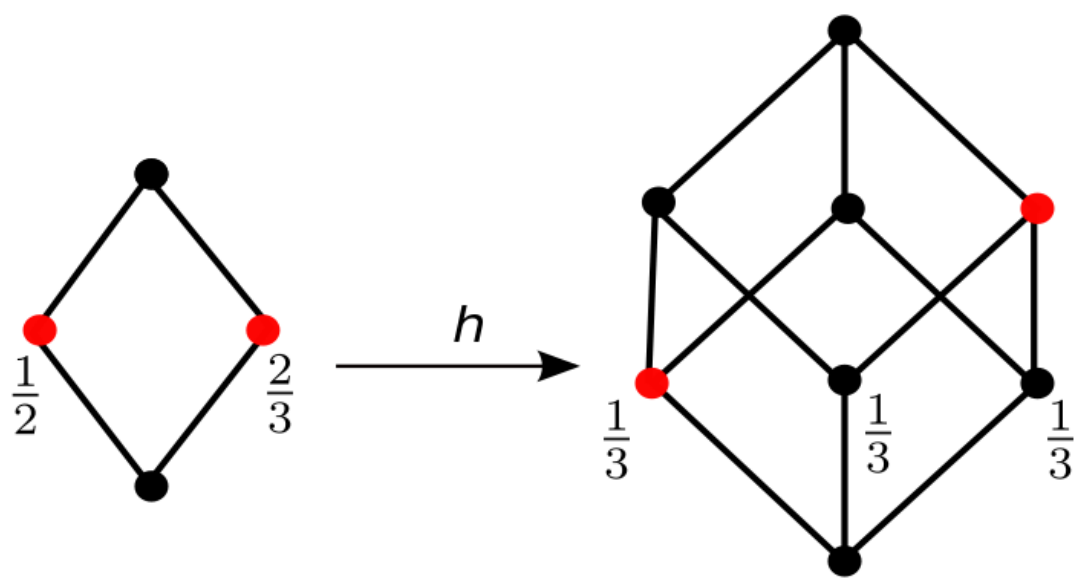

Here, we have a space with a non-uniform measure on the two atoms on the left, and its uniform-measure extension on the right. The image of one of 
the atoms of the left space through the homomorphism $h$ is still an atom, but the other atom (the one with probability $\frac{2}{3}$ ) loses this status; it lies above two atoms in the extension.

Now, consider an example in which the initial space contains a correlated pair of events without an SCC (it is the same space which Hofer-Szabó, Rédei \& Szabó [2000] present as not causally closed):

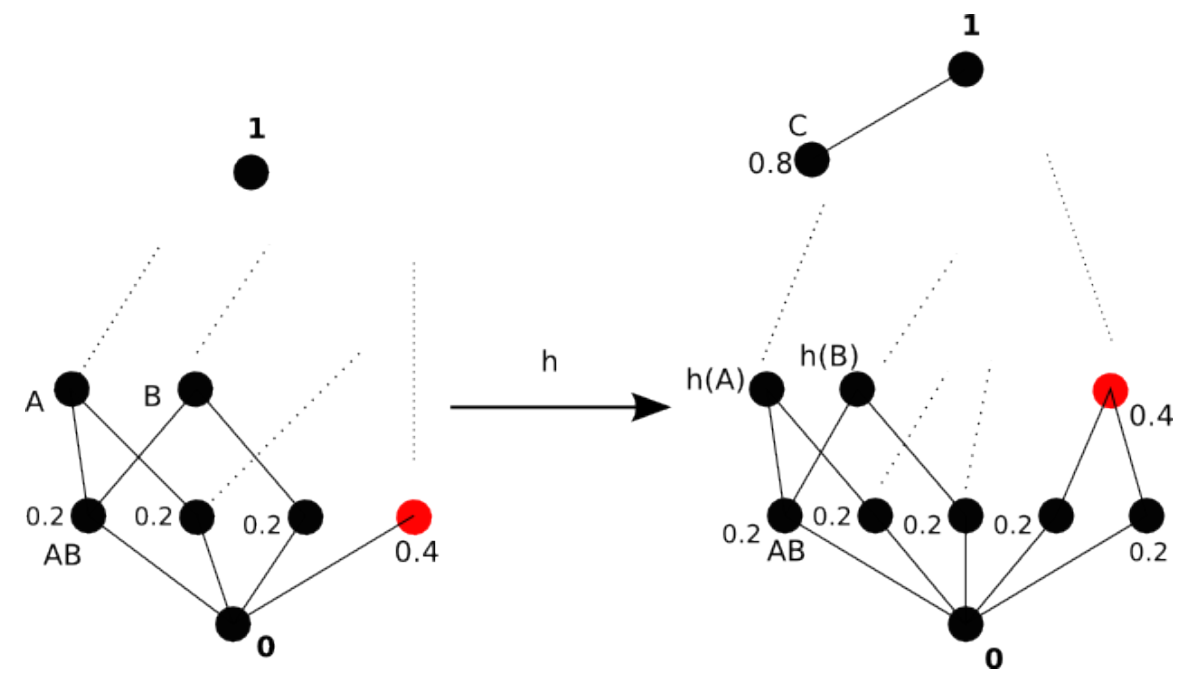

Events $A$ and $B$ are correlated in the left space, but lack an SCC in that space. Their images in the space on the right, however, possess an SCC (the event $C$ ), the construction of which was possible due to the fact that the atom with probability $\frac{2}{5}$ has been split into two atoms with probability $\frac{1}{5}$. This is the most fortunate case possible, since the space on the right is (as we know from the results by Gyenis \& Rédei [2004]) the only finite causally closed space (with no zero-probability atoms). In general, the product of the procedure of "splitting the atoms" will be a finite space with the uniform measure. But as far as explaining correlations is concerned, this is no worse, since (as we know from the results presented in the preceding chapter), all such spaces are causally up-to-3-closed (w.r.t. the relation of logical independence).

Let us put together the above considerations in the form of a theorem.

Theorem 1 Any finite probability space $\langle\mathcal{F}, P\rangle$ with rational probabilities on the atoms of $\mathcal{F}$ has an extension which is causally up-to-3-closed w.r.t. the relation of logical independence.

A sketch of the proof. The probabilities of the atoms $\left\{a_{0}, \ldots, a_{m}\right\}$ of $\mathcal{F}$ constitute a finite list $\left\langle p_{0}, \ldots, p_{m}\right\rangle$ of fractions. Calculate the lowest common denominator $D$ of these fractions. Let $\mathcal{F}^{\prime}$ be the Boolean algebra with $D$ atoms $\left\{b_{1}, \ldots, b_{D}\right\}$ and let $P^{\prime}$ be the uniform measure on $\mathcal{F}^{\prime}$. Transform all the fractions $\left\langle p_{0}, \ldots, p_{m}\right\rangle$ so that their denominator is $D$. Let $\left\langle n_{0}, \ldots, n_{m}\right\rangle$ be the list of numerators of the corresponding fractions from $\left\langle p_{0}, \ldots, p_{m}\right\rangle$. Of course, $\sum_{i=0}^{m} n_{i}=D$. Let $h: \mathcal{F} \rightarrow \mathcal{F}^{\prime}$ be the homomorphism which assigns to any 
atom $a_{i}$ of $\mathcal{F}$ the supremum of $n_{i}$ atoms of $\mathcal{F}^{\prime}$ in the following way:

$$
\begin{aligned}
h\left(a_{0}\right) & =\left\{b_{1}, \ldots, b_{n_{0}}\right\} ; \\
h\left(a_{1}\right) & =\left\{b_{n_{0}+1}, \ldots, b_{n_{0}+n_{1}}\right\} ; \\
& \ldots
\end{aligned}
$$

It is evident that $\left\langle\mathcal{F}^{\prime}, P^{\prime}\right\rangle$ is an extension of $\langle\mathcal{F}, P\rangle$ by means of the homomorphism $h$. And by fact 12 , this extension is causally up-to-3 closed w.r.t. the relation of logical independence.

\section{Causal completability of classical probability spaces - the general case}

One way of solving the general problem of causal completability with regard to any family of correlated events would be to show that any space possesses a causally closed extension. A result of Gyenis \& Rédei [2004], reproduced above as fact 11, states that all atomless spaces are causally closed. We simply need to find a way of extending an arbitrary space to an atomless space. This is done in the proof of theorem 2. Let us state the initial problem formally; it was posed in Hofer-Szabó et al. [1999] and Hofer-Szabó et al. [2000]:

Problem 16 (Causal completability of classical probability spaces) Let $\langle\Omega, \mathcal{F}, P\rangle$ be a probability space and $\mathcal{U} \subseteq \mathcal{F}^{2}$ be the (possibly infinite) family of all pairs of genuinely independent correlated events for which no statistical common cause in $\langle\Omega, \mathcal{F}, P\rangle$ exists. Is there an extension $\left\langle\Omega^{\prime}, \mathcal{F}^{\prime}, P^{\prime}\right\rangle$ given by an embedding $h$ of $\langle\Omega, \mathcal{F}, P\rangle$ such that for any $\langle D, E\rangle \in \mathcal{U}$, there exists in $\left\langle\Omega^{\prime}, \mathcal{F}^{\prime}, P^{\prime}\right\rangle$ a statistical common cause for the pair $\langle h(D), h(E)\rangle$ ?

In view of fact 11 , we can answer this problem by showing that any classical probability space is extendable to an atomless space. We will use the following lemma:

Lemma 17 Let $\langle\Omega, \mathcal{F}, P\rangle$ be a probability space and let $\langle[0,1], \mathcal{B}, L\rangle$ be the space of all Borel subsets of the $[0,1]$ segment, $L$ being the Lebesgue measure. Then the product space $\langle\Omega \times[0,1], \Sigma, \mu\rangle$ of the above two spaces is atomless.

The proof uses the technique from chapter 211M of Fremlin [2001]. For the details on the construction of $\Sigma$, the event $\sigma$-algebra of the product space, see e.g. chapter $I V .6$ of Feller [1968], vol. 2.

Proof: Let $E \in \Sigma, \mu(E)>0$. Let $f$ be a function from $\left[0, \frac{1}{2}\right]$ to $[0,1]$ given by the formula $f(a)=\mu\left(E \cap\left(\mathbf{1}_{\mathcal{F}} \times\left[\frac{1}{2}-a, \frac{1}{2}+a\right]\right)\right)$. Observe that if $a, b \in\left[0, \frac{1}{2}\right]$ and $a \leqslant b$, then $f(a) \leqslant f(b) \leqslant f(a)+\mu\left(\mathbf{1}_{\mathcal{F}} \times\left[\frac{1}{2}-b, \frac{1}{2}+b\right]\right)-\mu\left(\mathbf{1}_{\mathcal{F}} \times\left[\frac{1}{2}-a, \frac{1}{2}+a\right]\right)=$ $f(a)+p\left(\mathbf{1}_{\mathcal{F}}\right) \cdot L\left(\left[\frac{1}{2}-b, \frac{1}{2}+b\right]\right)-p\left(\mathbf{1}_{\mathcal{F}}\right) \cdot L\left(\left[\frac{1}{2}-a, \frac{1}{2}+a\right]\right)=f(a)+2 b-2 a$. Therefore, $f$ is continuous (as $b$ approaches $a, f(b)$ approaches $f(a)$ ).

Notice that $f(0)=0$ and $\lim _{n \rightarrow \frac{1}{2}} f(n)=\mu(E)>0$. Since we know that $f$ is continuous, we can apply the intermediate value theorem and conclude that for some $a \in\left(0, \frac{1}{2}\right), 0<f(a)<\mu(E)$. That is,

$$
0<\mu\left(E \cap\left(\mathbf{1}_{\mathcal{F}} \times\left[\frac{1}{2}-a, \frac{1}{2}+a\right]\right)\right)<\mu(E) .
$$


The event $E \cap\left(\mathbf{1}_{\mathcal{F}} \times\left[\frac{1}{2}-a, \frac{1}{2}+a\right]\right)$ is a subset of $E$ and has a strictly smaller measure. Since $E$ was arbitrary, $\langle\Omega \times[0,1], \Sigma, \mu\rangle$ is atomless. Q.E.D.

The following theorem gives a positive answer to problem 16 .

Theorem 2 Every probability space can be extended to a probability space which is causally closed.

Proof: Let $\langle\Omega, \mathcal{F}, p\rangle$ be a probability space. From lemma 17 we know that $\langle\Omega \times[0,1], \Sigma, \mu\rangle$, which is the product of $\langle\Omega, \mathcal{F}, p\rangle$ with the space of all Borel subsets of the $[0,1]$ interval with the Lebesgue measure, is atomless. Let $h: \mathcal{F} \rightarrow \Sigma$ be defined as $h(D)=D \times[0,1]$. It is immediate that $h$ is a Boolean algebra embedding of $\mathcal{F}$ into $\Sigma$, and so $\langle\Omega \times[0,1], \Sigma, \mu\rangle$ is an extension of $\langle\Omega, \mathcal{F}, p\rangle$. Moreover, from fact 11 we infer that it is a causally closed extension of $\langle\Omega, \mathcal{F}, p\rangle$. Q.E.D.

\section{Infinite statistical common cause systems for arbitrary pairs}

In this section we present our final result:

Theorem 3 Let $\mathbb{S}=\langle\Omega, \mathcal{F}, P\rangle$ be an atomless classical probability space.

Then all correlations of genuinely independent events in $\mathbb{S}$ have a countably infinite statistical common cause system.

The lengthy proof is relegated to Appendix A; below we recall the key construction of Wroński \& Marczyk [2010b] and sketch the way in which it can be used to establish the above theorem and a handful of corollaries.

The proof of the present theorem rests on two pillars: (1) a construction of a pair of correlated events together with an infinite SCCS which may be employed in an arbitrary atomless probability space and (2) the observation that in an atomless probability space every correlation can be supplied with a common cause included in the intersection of the correlated events. These are brought together by means of a certain quotient space construction.

Let us recall the basic infinite SCCS construction. Pictured below are two events $A$ and $B$ in the atomless probability space of all Borel subsets of the $[0,1)$ interval:

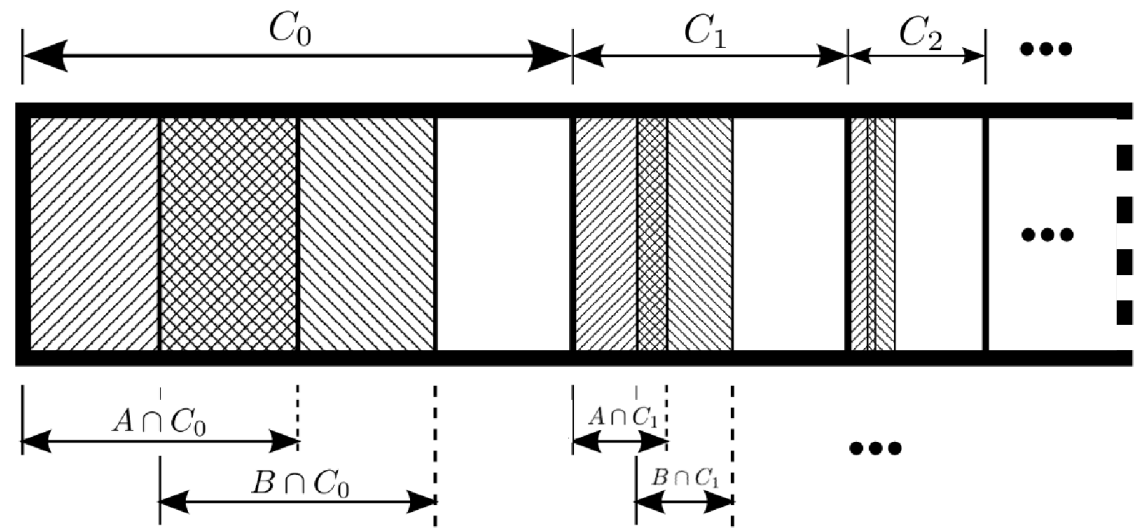

Originally appeared in Wroński \&6 Marczyk [2010b], reprinted with permission. 
The conditional probabilities of $A$ and $B$ given $C_{i}$ are both equal to $\frac{1}{2^{i+1}}$, while the conditional probability of their intersection given $C_{i}$ is $\frac{1}{\left(2^{i+1}\right)^{2}}$. Thus all the $C_{i}$ s are screeners-off for the pair and the statistical relevance condition is immediately seen to be satisfied. The fact that $A$ and $B$ are correlated then follows from the deductive property of SCCSs (as mentioned in section 2).

The intention of Wroński \& Marczyk [2010b] was to display an example of an infinite SCCS; thus the probability space in the context of which the construction was carried out and the correlated events for which the infinite SCCS is eventually supplied were also particular examples well-suited for this purpose. However, in light of fact 10, in the context of an atomless probability space the same basic construction can be used to construct an SCCS for a given pair of genuinely independent correlated events, as long as the probabilities of their intersection, both their differences and the complement of their union are appropriate (more specifically, the proportions between these probabilities are important; see fact 27 for details).

Further, it is readily apparent that the construction would work if the conditional probabilities of $A$ and $B$ given $C_{0}$ were both $\frac{1}{3}$, or indeed any other number less than 1 , as long as (1) the conditional probabilities of $A$ and $B$ given the $C_{i}$ s were always equal to the squares of the conditional probabilities of $A$ and $B$ given the $C_{i}$ s and (2) the statistical relevance condition was maintained by choosing, as $i$ increases, ever smaller conditional probabilities. (It is however not necessary to maintain any particular differences or ratios between the conditional probabilities at various $C_{i}$ s.) Finally, the construction would also work if we used a finite set of $C_{i} \mathrm{~s}$, as long as there were at least two, an observation leading directly to corollary 19.

Since our task is to provide an infinite SCCS for an arbitrary pair of genuinely independent events in an arbitrary atomless space, we cannot expect to be able to apply this basic construction directly. Happily, it is always possible to construct an SCCS comprising an event included in the intersection of the given correlated events (and thus trivially screening off their correlation), a single possibly non-trivial screener-off (in the sense that the conditional probabilities involving this screener-off will in general differ for the two given events) and an infinite collection of events chosen as in the basic infinite SCCS construction. Moreover, the conditional probabilities involving the events composing the latter collection can be chosen with a great degree of freedom (as they can in the basic infinite SCCS construction), although some care must be taken to ensure that none of them are greater than those involving the non-trivial screeneroff; the length of the proof results chiefly from the method in which a formula for calculating provably safe values for these conditional probabilities is proven correct.

Together with the above lemma 17, our theorem proves that any classical probability space can be extended to a space in which all correlations between genuinely independent events have a countably infinite common cause system, thus answering a question posed in Hofer-Szabó \& Rédei [2006]. Finally, the aforementioned freedom in choosing the conditional probabilities in the part of our construction resting on the basic infinite SCCS construction permits applying the same proof, with only minor modifications, to demonstrate the following two corollaries:

Corollary 18 Let $\mathbb{S}=\langle\Omega, \mathcal{F}, P\rangle$ be an atomless classical probability space. 
Then all correlations of genuinely independent events in $\mathbb{S}$ have uncountably many countably infinite statistical common cause systems.

Corollary 19 Let $\mathbb{S}=\langle\Omega, \mathcal{F}, P\rangle$ be an atomless classical probability space.

Then all correlations of genuinely independent events in $\mathbb{S}$ have, for any $n \in \mathbb{N}, n>2$, uncountably many statistical common cause systems of size $n$.

\section{Conclusion}

We are not aware of any open problems left regarding the explanation of correlations between events using Reichenbach-style notions in classical probability spaces. If anything, this paper shows that it is suprisingly easy to find such explanations, whether they should be statistical common causes or (even infinite) common cause systems. The ease of obtaining these may lead one to think that much "fleshing out" is due in order for this approach to move closer to a philosophically relevant account of causality. Attempting such a task would mean stepping outside the bounds of this technical article. Still, let us point to two ways in which one might continue the research. The first is to switch to the more general context of random variables, as opposed to events. Initial steps in this direction have been taken in Gyenis \& Rédei [2010]. The second would be to attempt to transfer the results to non-classical probability spaces.

\section{A Proof of theorem 3}

Before embarking on the proof of the above theorem, we will need to establish a handful of helpful facts.

Fact 20 A pair of events $\langle A, B\rangle$ is correlated iff

$$
P(A \cap B) P\left((A \cup B)^{\perp}\right)>P(A \backslash B) P(B \backslash A) .
$$

Proof: Observe that

- $P(A)=P(A \cap B)+P(A \backslash B)$;

- $P(B)=P(A \cap B)+P(B \backslash A)$;

- $P(A \backslash B)+P(A \cap B)+P(B \backslash A)+P\left((A \cup B)^{\perp}\right)=1$. 
The proof of the $(\Rightarrow)$ direction proceeds top-to-bottom below, while the $(\Leftarrow)$ direction is proven bottom-to-top:

$$
\begin{aligned}
P(A \cap B)> & P(A) P(B) \\
P(A \cap B)> & (P(A \backslash B)+P(A \cap B))(P(B \backslash A)+P(A \cap B)) \\
P(A \cap B)> & P(A \backslash B) P(B \backslash A)+(P(A \backslash B)+P(B \backslash A)) P(A \cap B)+ \\
& (P(A \cap B))^{2} \\
1> & P(A \backslash B)+P(B \backslash A)+P(A \cap B)+\frac{P(A \backslash B) P(B \backslash A)}{P(A \cap B)} \\
1-(P(A \backslash B)+P(B \backslash A)+P(A \cap B))> & \frac{P(A \backslash B) P(B \backslash A)}{P(A \cap B)} \\
P\left((A \cup B)^{\perp}\right)> & \frac{P(A \backslash B) P(B \backslash A)}{P(A \cap B)} \\
P(A \cap B) P\left((A \cup B)^{\perp}\right)> & P(A \backslash B) P(B \backslash A)
\end{aligned}
$$

(In both directions we divide by $P(A \cap B)$. In the $(\Rightarrow)$ direction it has to be nonzero since the assumption is that $A$ and $B$ are correlated. In the $(\Leftarrow)$ direction we can also assume that $P(A \cap B)>0$ : otherwise it would not be the case that $P(A \cap B) P\left((A \cup B)^{\perp}\right)>P(A \backslash B) P(B \backslash A)$. $) \quad$ Q.E.D.

Fact 21 Every pair of genuinely independent correlated events in an atomless classical probability space has a statistical common cause contained in the intersection of the two events.

Proof: Let $\langle A, B\rangle$ be a pair of genuinely independent correlated events in an atomless classical probability space whose probability measure is called $P$.

Then for any non-empty event $C \subseteq A \cap B$, all the requirements of the definition of a common cause which do not mention $C^{\perp}$ are satisfied trivially. Thus, our task is to find an appropriate $C^{\perp} \supseteq(A \cap B)^{\perp}$ to satisfy the remaining requirements.

To this end, note that as long as our chosen $C^{\perp}$ has the property that both $P\left(A \mid C^{\perp}\right)$ and $P\left(B \mid C^{\perp}\right)$ are less than 1, we need not worry about the statistical relevance condition. (It will be trivially satisfied, since for $C \subseteq A \cap B$, $P(A \mid C)=P(B \mid C)=1$.)

It follows that any screener-off containing $(A \cap B)^{\perp}$ (except the unit of the space) is capable of filling the role of $C^{\perp}$. By our hypothesis regarding $\langle A, B\rangle$ and fact 20 ,

$$
P(A \cap B) P\left((A \cup B)^{\perp}\right)>P(A \backslash B) P(B \backslash A) .
$$

Since the space in question is atomless (see def. 9), we can partition $A \cap B$ into two non-overlapping events $C$ and $D$, such that

$$
P(D) P\left((A \cup B)^{\perp}\right)=P(A \backslash B) P(B \backslash A) ;
$$

then $C$ is the required common cause and $C^{\perp}=D \cup(A \backslash B) \cup(B \backslash A) \cup(A \cup B)^{\perp}$, its complement, is a screener-off satisfying all the requirements on the complement of a common cause set down in definition 5. Q.E.D.

The following parameters are crucial to the construction of the infinite SCCS to be undertaken below: 
Definition 22 Given an atomless classical probability space $\mathbb{S}$ and two genuinely independent correlated events $A$ and $B$, define

$$
\begin{aligned}
M_{\mathbb{S}} & :=\min \left\{P\left(A \mid C^{\perp}\right), P\left(B \mid C^{\perp}\right)\right\} \\
m_{\mathbb{S}} & :=\max \left\{\frac{1}{n} \mid n \in \mathbb{N}, \frac{1}{n}<M_{\mathbb{S}}\right\},
\end{aligned}
$$

for $C^{\perp}$ constructed as in the proof of fact 21 .

Definition 23 Let $\mathbb{S}=\langle\Omega, \mathcal{F}, P\rangle$ be a classical probability space and $E \neq \Omega$ an event in that space.

Set

$$
\begin{aligned}
\Omega^{\prime} & :=\Omega \backslash E \\
\mathcal{F}^{\prime} & :=\{X \backslash E \mid X \in \mathcal{F}\} \\
P^{\prime}(X) & :=\frac{P(X)}{P\left(E^{\perp}\right)}, \quad \text { for } X \in \mathcal{F}^{\prime} .
\end{aligned}
$$

Then $\mathbb{S} /_{E}:=\left\langle\Omega^{\prime}, \mathcal{F}^{\prime}, P^{\prime}\right\rangle$ is a probability space (a claim easily verifiable by the Reader) and may be referred to as the space arising from $\mathbb{S}$ by factoring out the event $E$, the quotient space of $\mathbb{S}$ determined by $E$ etc.

The notation $A / E$, where $A$ and $E$ are events, will be used to refer to the set $A \backslash E$ as an event in the appropriate quotient space.

Notice that $P^{\prime}(X)=P\left(X \mid E^{\perp}\right)$.

Fact 24 The field of sets $\mathcal{F}^{\prime}$ from definition 23 is isomorphic to the quotient algebra $\mathcal{F} / \Theta(\emptyset, E)$, where $\Theta(\emptyset, E)$ is the smallest congruence with respect to which $\emptyset$ and $E$ are equivalent. The division operator represents here the usual quotient algebra construction on Boolean algebras. Q.E.D.

Fact 25 Consider an atomless classical probability space.

For any $\epsilon>0$, there exists a $\delta>0$ such that factoring out an arbitrary event $E$ of measure less than $\delta$ results in a quotient space such that for any event $A$ from the original space,

$$
1-\epsilon<\frac{P(A)}{P^{\prime}(A / E)}<1+\epsilon
$$

where $P$ and $P^{\prime}$ are the probability measures in the original space and the quotient space, respectively.

Proof: This follows immediately from the definition of the probability measure in the quotient space in definition 23. Q.E.D.

Fact 26 Consider an atomless classical probability space $\mathbb{S}$ and a pair of genuinely independent events $A, B$ in $\mathbb{S}$. Then there exists a $\delta>0$ such that for any classical probability space $\mathbb{S}^{\prime}$ arising from $\mathbb{S}$ by factoring out an event of measure no greater than $\delta$ from each of the events $A \backslash B, A \cap B, B \backslash A,(A \cup B)^{\perp}$, $m_{\mathbb{S}^{\prime}} \geqslant m_{\mathbb{S}}$ 
Proof: First, we need to ensure that $m_{\mathbb{S}}<M_{\mathbb{S}^{\prime}}$. To this end, notice that $M_{\mathbb{S}}$ is uniquely determined by the probabilities of the events $A \backslash B, A \cap B$, $B \backslash A$ and $(A \cup B)^{\perp}$, and by fact 25 we may choose a $\delta$ so that factoring out an arbitrary event $E$ of measure at most $4 \delta$ from $\mathbb{S}$ has an arbitrarily small impact on the probabilities of $A \backslash B / E$ etc. In particular, we may choose an event whose intersection with each of the events $A \backslash B$ etc. has measure at most $\delta$.

Second, notice that by definition $22 m_{\mathbb{S}^{\prime}}=\max \left\{\frac{1}{n} \mid n \in \mathbb{N}, \frac{1}{n}<M_{\mathbb{S}^{\prime}}\right\}$. Since we already know that $m_{\mathbb{S}}<M_{\mathbb{S}^{\prime}}$, it follows that $m_{\mathbb{S}^{\prime}} \geqslant m_{\mathbb{S}}$. Q.E.D.

Fact 27 Suppose $\left\{C_{i}\right\}_{i \in I}$ is an infinite SCCS for a pair of events $A, B$ in a probability space $\mathbb{S}$ constructed according to the method from Wronski \& Marczyk [2010b] with the construction starting at probability $\frac{1}{d}, d \in \mathbb{N}$. Then the probabilities of $A$ and $B$ in $\mathbb{S}$ are as follows:

$$
P(A)=P(B)=\sum_{n \in \mathbb{N}} \frac{1}{d+n} \frac{1}{2^{n+1}},
$$

whereas the probability of $(A \cup B)^{\perp}$ is

$$
P\left((A \cup B)^{\perp}\right)=\sum_{n \in \mathbb{N}} \frac{d+n-1}{d+n} \frac{1}{2^{n+1}} .
$$

The probability of $A \cap B$ is

$$
P(A \cap B)=\sum_{n \in \mathbb{N}} \frac{1}{(d+n)^{2}} \frac{1}{2^{n+1}} .
$$

Proof: Immediate from the relevant definitions. Q.E.D.

The above formulae determine the proportions between the probabilities of $A \backslash B, A \cap B, B \backslash A,(A \cup B)^{\perp}$ in $\mathbb{S}$. These will be the proportions between the probabilities of the events factored out from the given space in the proof below.

We are now prepared to prove theorem 3:

Theorem 3 Let $\mathbb{S}=\langle\Omega, \mathcal{F}, P\rangle$ be an atomless classical probability space.

Then all correlations of genuinely independent events in $\mathbb{S}$ have a countably infinite statistical common cause system.

Proof: Consider an atomless classical probability space $\mathbb{S}$ and a pair of correlated, genuinely independent events $A, B$ in $\mathbb{S}$.

By fact 26 , it is possible to pick a $\delta$ such that factoring out events of measure at most $\delta$ (let us call them small events) from each of the events $A \backslash B, A \cap B$, $B \backslash A$ and $(A \cup B)^{\perp}$ results in a quotient space whose $m$ parameter (see definition 22 ) is no smaller. In particular, we may pick the measures of the events we factor out from each part of the space so that the proportions of their measures are as required by fact 27 with $d=m_{\mathbb{S}}^{-1}$.

We may then partition the union of the small events we factored out so as to recreate the infinite SCCS construction within their union, even as we partition the remainder of the space as in the regular common cause construction with the common cause $C$ contained in the intersection of $A$ and $B$ and its "complement" $D$ equal to the difference of the unit of the space and the union of $C$ and the 
small events. Notice that factoring out the small events does not decrease the $m$ parameter: $m_{\mathbb{S}^{\prime}} \geqslant m_{\mathbb{S}}$. Therefore for each event $E$ belonging to the recreation of the infinite SCCS construction inside the union of the factored out small events we get that $P(A \mid D)>P(A \mid E)$ and $P(B \mid D)>P(B \mid E)$, thus securing the statistical relevance condition. Therefore this infinite ensemble whose union is the union of the small events, together with $C$ and $D$, constitues an infinite SCCS for $A$ and $B$ in the original space $\mathbb{S}$. Q.E.D.

Proof: [of corollary 18]

Immediate from the proof of theorem 3: observe that the recreation of the infinite SCCS construction may start from any positive real number less than $m_{\mathbb{S}}$. Q.E.D.

Proof: [of corollary 19]

Again, observe that the recreation of the infinite SCCS construction may start from any positive real number less than $m_{\mathbb{S}}$ and then terminate after any finite number of steps. Q.E.D.

Formalising the above two proofs would require a straightforward generalisation of fact 27 .

\section{References}

Arntzenius, F. (1992). The Common Cause Principle. PSA: Proceedings of the Biennial Meeting of the Philosophy of Science Association, Vol. 1992, Volume Two: Symposia and Invited Papers.

Beebee, H. (1998). Do causes raise the chances of effects? Analysis, 58(3), $182-190$.

Feller, W. (1968). An Introduction to Probability Theory and its Applications. John Wiley \& Sons, Inc.

Fremlin, D. (2001). Measure Theory, volume 2. Torres Fremlin.

Gyenis, B. \& Rédei, M. (2004). When Can Statistical Theories be Causally Closed? Foundations of Physics, 34(9), 1284-1303.

Gyenis, B. \& Rédei, M. (2010). Causal completeness in general probability theories. In M. Suárez (Ed.), Probabilities, Causes, and Propensities in Physics. Synthese Library, Springer.

Hofer-Szabó, G. \& Rédei, M. (2004). Reichenbachian Common Cause Systems. International Journal of Theoretical Physics, 43(7/8), 1819-1826.

Hofer-Szabó, G. \& Rédei, M. (2006). Reichenbachian Common Cause Systems of arbitrary finite size exist. Foundations of Physics, 36(5), 745-756.

Hofer-Szabó, G., Rédei, M., \& Szabó, L. E. (1999). On Reichenbach’s common cause principle and Reichenbach's notion of common cause. The British Journal for the Philosophy of Science, 50(3), 377-399.

Hofer-Szabó, G., Rédei, M., \& Szabó, L. E. (2000). Reichenbach’s Common Cause Principle: Recent Results and Open Questions. Reports on Philosophy, 20, 85-107. 
Pearl, J. (2000). Causality. Models, Reasoning, and Inference. Cambridge University Press.

Rédei, M. \& Summers, S. (2002). Local primitive causality and the common cause principle in quantum field theory. Foundations of Physics, 32(3), 335355 .

Reichenbach, H. (1971). The Direction of Time. University of California Press. Reprint of the 1956 edition.

Sober, E. (2001). Venetian Sea Levels, British Bread Prices, and the Principle of the Common Cause. The British Journal for the Philosophy of Science, 52, 331-346.

van Fraassen, B. C. (1982). The Charybdis of Realism: Epistemological Implications of Bell's Inequality. Synthese, 52, 25-38. Reprinted with additions in Cushing, JT., McMullin, E. (eds) (1989).

Williamson, J. (2005). Bayesian Nets and Causality. Philosophical and Computational Foundations. Oxford University Press.

Wroński, L. (2010). The Common Cause Principle. Explanation via screening off. PhD thesis, Jagiellonian University, Kraków. Archived at http://jagiellonian.academia.edu/LeszekWroński.

Wroński, L. \& Marczyk, M. (2010a). A New Notion of Causal Closedness. Under review.

Wroński, L. \& Marczyk, M. (2010b). Only Countable Reichenbachian Common Cause Systems Exist. Foundations of Physics, 40, 1155-1160. 